\title{
Determinantes de la innovación en empresas constructoras de la Región de Atacama, Chile
}

\author{
Determinants of innovation in construction firms in the Atacama Region, Chile
}

\section{Hernán Pape ${ }^{1}$ y Amin Nazer ${ }^{2}$}

Fecha de entrega: 27 de octubre 2020 Fecha de aceptación: 1 de junio 2021

${ }^{1}$ Departamento de Industria y Negocios, Universidad de Atacama, Avenida Copayapu 485, Copiapó, Chile, hernan.pape@uda.cl

${ }^{2}$ Departamento de Construcción, Universidad de Atacama, Avenida Copayapu 485, Copiapó, Chile, amin.nazer@uda.cl

La innovación es un proceso que debe obedecer a una planificación de tipo estratégica en la empresa y juega un rol de importancia al momento de competir con otros productores. La innovación no se ha investigado con rigurosidad en las PYME (pequeña y mediana empresa) del sector construcción a nivel regional en Chile, excepto en la capital del país. Por ello, el objetivo del presente artículo fue identificar las unidades más factibles para innovar y valorar los principales factores que favorecen y desfavorecen la instalación de la innovación en el sector construcción en la región de Atacama, evaluando estadísticamente la relación entre los factores de innovación. Se consideró una muestra no probabilística compuesta por 12 empresas PYME, aplicando un cuestionario con preguntas cerradas. Los resultados indican que la totalidad de los expertos encuestados opina que la innovación es una herramienta estratégica muy relevante para mejorar la competitividad de las empresas constructoras de la región. Además, se detectó que las unidades más factibles para innovar son: materiales, productos, equipos y máquinas. Asimismo, las áreas de procesos y estructura organizacional fueron señaladas como relevantes para la instalación de la innovación en la empresa. Los factores que favorecen en mayor medida la innovación son: un buen entorno económico, profesionales preparados para innovar y disponibilidad de recursos económicos. Por otra parte, los factores que desfavorecen la instalación de la innovación son: la falta de cultura de innovación, la estructura organizacional poco flexible, la escasez de recursos y la baja capacidad gerencial.

Palabras clave: factores de innovación, sector construcción, Pymes, proceso de innovación
Innovation is a process that is due to strategic planning in the company and it plays an important role when competing with other producers. Considering that innovation has not been rigorously investigated in SMEs (small med-size enterprises) in the construction sector at the regional level in Chile, except in the capital of the country. The objective of this paper was to evaluate the main factors that favour and disadvantage the installation of innovation in the construction sector in the Atacama region, Chile, statistically evaluating the relationship between innovation factors. A nonprobabilistic sample composed of 12 SMEs companies was considered, applying a questionnaire with open and closed questions oriented. The results indicate that all of the experts surveyed believe that innovation is a very relevant strategic tool to improve the competitiveness of construction companies in the region. In addition, it was detected that the feasible areas to innovate are: materials, products, equipment and machines. On the other hand, the areas of processes and organizational structure were indicated as relevant for the installation of innovation in the company. The factors that favour innovation are: a good economic environment, professionals prepared to innovate and availability of economic resources. Moreover, the factors that hinder the installation of innovation are: the lack of an innovation culture, the rigid organizational structure, the scarcity of resources and the low management capacity.

Keywords: innovation determinants, construction sector, SME, process innovation

\section{Introducción}

Cada día la competencia es más fuerte entre las empresas que participan en los diferentes sectores de la economía de una nación. Los clientes son más sofisticados y exigentes con los satisfactores de sus necesidades. El sector construcción no está ajeno a esta realidad. Por ello, las 
empresas constructoras, y también aquellas afines a esta actividad, buscan mejorar su posicionamiento competitivo, ya sea mejorando sus procesos o diferenciando sus ofertas de productos a través de diversas estrategias que incluye nuevos productos, reestructuración organizacional, marketing, capacitación, jugando la innovación un rol importante en todos ellos.

Un concepto relevante dentro del ámbito de la competitividad se puede relacionar con la gestión de la innovación en la empresa constructora. Para la CDT (2012), la gestión de la innovación "es la organización y dirección de los recursos tanto humanos como económicos, con el fin de aumentar la creación de nuevos conocimientos, la generación de ideas que permitan obtener nuevos productos, procesos y servicios o mejorar los ya existentes, y la transferencia de esas mismas ideas a las fases de producción, construcción o cualquier otro proceso según el ámbito de acción de cada compañía".

Como se ha descrito, el concepto de innovación está en el entorno del desarrollo empresarial, y ésta puede medirse en términos de su instalación a nivel de país. Según GII (2019), Chile se ubica en el puesto 51 del ranking mundial de innovación del año 2019, clasificándose también, como un país débil en materia de ingresos. Por otra parte, ocupa el puesto 64 en el número de solicitudes de patentes residentes presentadas en una oficina de patentes nacional o regional determinada, no obstante, en este aspecto es el mejor posicionado entre los países sudamericanos. Respecto del indicador cantidad de solicitudes de diseño industrial presentadas en una oficina nacional o regional determinada, Chile se ubica débilmente en el puesto 105, a la zaga de varios países sudamericanos. Al respecto, se hace necesario evaluar los factores que favorecen y aquellos que desfavorecen la instalación y el desarrollo de la innovación en el sector de la construcción.

Pero, en particular, ¿qué se debe entender como innovación en el sector de la construcción? En términos generales, el Diccionario de la Lengua Española de la Real Academia Española define innovación como la creación o modificación de un producto, y su introducción en un mercado; mientras que para la CORFO (2017), con una visión más amplia, la define como la creación de valor a través de la transformación de ideas o conocimientos en un producto, bien o servicio nuevo o mejorado. La OCDE clasifica las innovaciones como técnicas, orientada a productos y procesos, o no técnicas, referidas a cambios en la estructura organizativa, la cultura, las técnicas de gestión y la dirección estratégica (Blayse y Manley, 2004).

Asimismo, existen fuentes de financiamiento para el apoyo de la innovación en la empresa. Una vía de acceso a recursos económicos es a través de fondos gubernamentales que CORFO tiene disponible para la presentación de proyectos a diferentes programas: Bienes Públicos, Centros Fortalece PYME, Súmate a Innovar, Súmate a Innovar en Ecodiseño, Innova Región, Crea y Valida, Innova Alta Tecnología y, Consolida y Expande. En este sentido, la región de Atacama tiene un bajo indicador de proyectos postulados durante el año 2019 (en todas las áreas), alcanzando sólo $0.85 \%$ respecto del total nacional y un $1.19 \%$ de proyectos finalmente adjudicados en algún programa. Sin embargo, las estadísticas muestran que no fue adjudicado ningún proyecto del sector de la construcción, lo que podría ser debido al desinterés o desconocimiento de las empresas por acceder a estos fondos para la innovación. Además, CDT (2010) ha estudiado la instalación y desarrollo de una estrategia de innovación al interior de empresas constructoras ubicadas en la Región Metropolitana; sin embargo, no se han reportado estudios en otras regiones del país, que permitan evaluar los factores locales. Por tanto, el objetivo del presente estudio fue identificar las potenciales áreas de una empresa constructora donde innovar y valorar los principales factores, ya sea endógeno o exógeno, que favorecen y desfavorecen la instalación de la innovación en el sector construcción en la región de Atacama. Dado lo anterior, parece interesante preguntarse si las empresas del sector construcción de la región de Atacama están conscientes de la importancia estratégica de la innovación, como factor o herramienta de competitividad. Por ello, esta investigación tiene los siguientes objetivos específicos:

1. Determinar si las empresas constructoras de Atacama reconocen a la innovación como una herramienta estratégica para mejorar su competitividad.

2. Identificar las unidades o ámbitos al interior de una empresa constructora más pertinentes para innovar.

3. Valorar las variables internas o externas que favorecen y desfavorecen la innovación en el sector construcción.

4. Medir la relación entre los factores de innovación a 
través de un análisis correlacional.

5. Efectuar un análisis de varianza (ANOVA) para comprobar si existe alguna unidad preferida para innovar dentro de la empresa constructora.

6. Efectuar un análisis de varianza (ANOVA) para determinar si existe una diferencia significativa en la valoración de los diferentes factores que favorecen la innovación.

7. Encontrar modelos de regresión lineal múltiple que permitan explicar la relación entre factores que favorecen la innovación.

Un interesante estudio sobre la instalación de la gestión de innovación en la empresa ha sido realizado por la CDT (2010), a siete destacadas empresas constructoras chilenas, todas establecidas en la Región Metropolitana. El estudio concluyó que para desarrollar adecuadamente la innovación al interior de ellas, se deben considerar aspectos tales como: incorporar la innovación a nivel estratégico, estructurar la organización de la empresa para la innovación, propiciar el cambio cultural hacia la innovación, desarrollar proyectos de innovación acorde con los objetivos y las capacidades de la empresa, identificar las ideas innovadoras realizadas en obra y difundirlas al interior de la organización $\mathrm{y}$, rescatar efectivamente el máximo beneficio de la vigilancia tecnológica. Por otra parte, el mismo estudio declara que la mayoría de las empresas constructoras del país no consideran atractivas las inversiones en $\mathrm{I}+\mathrm{D}+\mathrm{i}$ porque no han entendido su papel como factor clave de competitividad. A lo anterior, se suma que las políticas de fomento en Latinoamérica no consideran a la empresa como el foco del proceso de innovación (Heitor et al., 2014). Otros factores que inhiben la inversión son la presión en los plazos de ejecución de los proyectos, la competencia por la mejor oferta y, la participación de otros actores externos a la empresa, tales como los mandantes públicos, promotores privados, empresas consultoras, subcontratistas, proveedores, entre otros, los cuales incorporan variables que la empresa constructora principal no puede controlar.

Hoy en día, se plantea que la innovación es un proceso que debe obedecer a una planificación de tipo estratégica en la empresa de un determinado sector productivo de bienes y servicios, y ésta juega un rol de importancia al momento de competir con otros oferentes. La innovación está en el corazón de la economía moderna basada en el conocimiento (OCDE, 2005; EC, 2010) y difiere en cada sector, es así como patrones de innovación en la manufactura difieren de los servicios (DTI, 2007) como también, si se aplica a un proyecto internacional, nacional o regional (Aouad, 2010). Algunos estudios han enfatizado las diferencias regionales en la innovación y el desempeño de las empresas (Hoogstra y van Dijk, 2004; Hölzl, 2009; Mendonça et al., 2004), sin embargo, para otros autores, este factor no es relevante (Littunen y Tohmo, 2003; North y Smallbone, 2000).

Otro aspecto de importancia a considerar es la interacción de las empresas constructoras con otros actores para desarrollar e implementar nuevas soluciones (Bygballe e Ingemansson, 2014). La clave es que existe una correlación positiva entre la innovación y el crecimiento (Heimonen, 2012). Además, se reportan estudios sobre estrategias para implementar la innovación en proyectos de construcción (Slaughter, 2000; Winch; 2003) y cómo las empresas constructoras gestionan el proceso de innovación basándose en modelos conceptuales (Seaden y Manseau, 2001; Dikmen et al., 2005) y estudios de casos (Koskela y Vrijhoef, 2001; Sexton y Barrett, 2003; Cleasby, 2004), todos asociados a estructuras organizacionales diferentes de la industria de la construcción nacional.

En la Tabla 1, se presentan los temas abordados y áreas relacionadas con la empresa y las referencias, las cuales permitirán establecer las variables a investigar en el presente estudio.

\section{Metodología}

Para investigar sobre los factores de la innovación en la construcción, se planteó la siguiente hipótesis: La innovación es una herramienta estratégica muy relevante para mejorar la competitividad de las empresas constructoras. El desarrollo del estudio focalizó empresas PYME del sector construcción de Atacama. Según una clasificación del Ministerio de Economía (BCN, 2013), una empresa pequeña es la que al año vende productos o servicios por valores entre 2400 y 25 mil UF (US\$ 98.330 y US\$ 1.024.275) y una empresa mediana vende entre 25 mil y 100 mil UF (US\$1.024.275 y US\$ 4.097.098).

El estudio fue de tipo cuantitativo, correlacional y 
Tabla 1: Área de empresa y temas abordados según diversas fuentes

\begin{tabular}{|c|c|c|}
\hline Área de empresa & Temas abordados & Referencia \\
\hline \multirow{5}{*}{ Economía y finanzas } & Mejora del nivel financiero & Abdel-Razek (1998) \\
\hline & Incentivos, utilidad percibida & Talukder (2012) \\
\hline & $\begin{array}{l}\text { Recompensas por innovación, amortización } \\
\text { Período de recuperación estimado }\end{array}$ & Goodrum y Haas (2000) \\
\hline & Recursos de la empresa & Hardie y Newell (2011) \\
\hline & Inversión en I+D & $\begin{array}{l}\text { Dubois y Gadde (2002) } \\
\text { Hardie y Newell (2011) } \\
\text { Heimonen (2012) } \\
\text { Manley y Mcfallan (2006) }\end{array}$ \\
\hline \multirow{7}{*}{ Personas y organización } & $\begin{array}{l}\text { Soporte propietario/cliente, } \\
\text { cultura organizacional y la comunicación }\end{array}$ & Gambatese y Hallowell (2011) \\
\hline & Apoyo gerencial & $\begin{array}{l}\text { Talukder (2012) } \\
\text { Goodrum y Haas (2000) } \\
\text { Gambatese y Hallowell (2011) } \\
\end{array}$ \\
\hline & Liderazgo, experimentar la diversidad & Goodrum y Haas (2000) \\
\hline & Imagen & Talukder (2012) \\
\hline & Influencia del cliente y del usuario final & Hardie y Newell (2011) \\
\hline & Comunicación & Goodrum y Haas (2000) \\
\hline & $\begin{array}{l}\text { Atmósfera de competición, tolerancia } \\
\text { Motivación por la innovación } \\
\text { Actitud hacia el cambio }\end{array}$ & $\begin{array}{l}\text { Goodrum y Haas (2000) } \\
\text { Talebi et al. }(2012)\end{array}$ \\
\hline \multirow{4}{*}{ Producción y calidad } & Uso de nuevas técnicas de construcción & $\begin{array}{l}\text { Cheung et al. (2004) } \\
\text { Luu et al. (2008) }\end{array}$ \\
\hline & Interés del cliente & Abdel-Razek (1998) \\
\hline & $\begin{array}{l}\text { Participación en el diseño } \\
\text { Nuevos productos y procesos }\end{array}$ & $\begin{array}{l}\text { Goodrum y Haas (2000) } \\
\text { Gledson y Phoenix (2017) }\end{array}$ \\
\hline & Condiciones basadas en proyectos & $\begin{array}{l}\text { Gledson y Phoenix (2017) } \\
\text { Hardie y Newell (2011) } \\
\text { Dubois y Gadde (2002) } \\
\text { Manley y Mcfallan (2006) }\end{array}$ \\
\hline $\begin{array}{l}\text { Gestión comercial y } \\
\text { ventas }\end{array}$ & $\begin{array}{l}\text { Formación, innovación personal } \\
\text { Imagen, interés del cliente } \\
\text { Influencias del cliente y usuario final }\end{array}$ & $\begin{array}{l}\text { Talukder (2012) } \\
\text { Gledson y Phoenix (2017) } \\
\text { Hardie y Newell (2011) } \\
\end{array}$ \\
\hline \multirow{3}{*}{ Tamaño y entorno } & Redes industriales & Hardie y Newell (2011) \\
\hline & Vinculación industria-universidad & $\begin{array}{l}\text { Talebi et al. (2012) } \\
\text { Aouad et al. (2010) }\end{array}$ \\
\hline & $\begin{array}{l}\text { Etapa de la industria, demanda } \\
\text { Tamaño y la edad de la empresa }\end{array}$ & Talebi et al. (2012) \\
\hline
\end{tabular}


descriptivo. La estructura de los datos es de tipo seccional porque ellos fueron tomados en un momento del tiempo. Se utilizó un muestreo por conveniencia, no probabilístico, ya que del universo de empresas solo se convocó a participar a 40 empresas asociadas a la Cámara Chilena de la Construcción CChC de la delegación Copiapó. Como resultado de la convocatoria, finalmente, participaron los representantes de doce empresas, constituyendo así, la muestra de la investigación. Se consideró una categorización según la actividad principal que la empresa desarrolla habitualmente, siendo seis de ellas especialistas en infraestructura, una en edificación, una en urbanización, una del sector inmobiliario, una empresa comercializadora de materiales de construcción, una con actividad de arriendo y venta de máquinas y equipos, y otra relacionada con servicios al sector de la construcción. A partir de los estudios presentados en la Tabla 1 y considerando los temas abordados por cada uno de ellos, se efectuó una clasificación que entregó como resultado seis áreas de interés de la empresa, y a partir de éstas se definieron las variables de innovación en la empresa constructora, las cuales se presentan en la Tabla 2.
Las variables consideradas en la investigación son las de unidades factibles de innovación, factores que favorecen la innovación y factores que la desfavorecen. La encuesta se estructuró en dos partes, la primera de ellas colectó datos de la empresa, tales como el área de especialización, antigüedad en el mercado regional, rango de ventas anuales, nivel de formación profesional del gerente o administrador y la cantidad de trabajadores. La segunda parte de la encuesta se orientó a la medición de las variables de innovación, considerando aspectos tales como la unidad de innovación, los factores que favorecen la innovación y los factores que no favorecen la innovación. Todas las preguntas del cuestionario fueron cerradas porque los encuestados respondieron mediante la selección de opciones establecidas. En el Anexo se presenta el cuestionario aplicado. Asimismo, se procedió a determinar la consistencia interna del instrumento utilizando el coeficiente de confiabilidad alfa de Cronbach, dando como resultado 0.648; a partir de este valor y considerando el trabajo investigativo de Arévalo y Padilla (2016), que describe escalas de clasificación de los niveles de fiabilidad al utilizar el Alfa de Cronbach, que se muestra

Tabla 2: Áreas de la empresa y variables relacionadas con la innovación

\begin{tabular}{|l|l|l|l|}
\hline \multirow{2}{*}{ Áreas } & \multicolumn{3}{|c|}{ Variables } \\
\cline { 2 - 5 } & Unidad de innovación & \multicolumn{1}{|c|}{ Factores que favorecen } & Factores que desfavorecen \\
\hline \multirow{2}{*}{ Personomía y finanzas } & Financiamiento & $\begin{array}{l}\text { Buena situación económica de entorno } \\
\text { Disponibilidad de recursos económicos }\end{array}$ & Escasez de recursos económicos de financiamiento externo \\
\hline Producción y calidad & $\begin{array}{l}\text { Estructura } \\
\text { organizacional }\end{array}$ & Pultura de innovación & Baja capacidad gerencial \\
\hline Gestión comercial y ventas & Procesos & Fomentar la capacitación & Falta de confianza de directivos \\
& Materiales & Interés en servicios de calidad & Poca motivación de directivos \\
\hline Tamaño y entorno & marketing & Contar con precios competitivos & Falta de cultura de innovación \\
\hline
\end{tabular}


en la Tabla 3, se concluyó que el valor obtenido indica que el instrumento tiene un buen nivel de fiabilidad.

Tabla 3: Clasificación de los niveles de fiabilidad según el Alfa de Cronbach

\begin{tabular}{|c|c|c|}
\hline Índice & Nivel de fiabilidad & Valor de Alfa de Cronbach \\
\hline 1 & Excelente & $0.9-1.0$ \\
\hline 2 & Muy bueno & $0.7-0.9$ \\
\hline 3 & Bueno & $0.5-0.7$ \\
\hline 4 & Regular & $0.3-0.5$ \\
\hline 5 & Deficiente & $0.0-0.3$ \\
\hline
\end{tabular}

Las percepciones de los expertos fueron recogidas aplicando una escala de Likert, se evaluó el grado de acuerdo utilizando las alternativas Muy bajo acuerdo (MB), Bajo acuerdo (B), Mediano acuerdo (M), Alto acuerdo (A), Muy alto acuerdo (MA). Posteriormente, las respuestas fueron tabuladas en tablas de frecuencia y se aplicaron funciones de estadística descriptiva.

Con los resultados obtenidos, se efectúa un análisis correlacional con la finalidad de medir el grado de relación entre las unidades y factores de innovación. También se presentan dos análisis de varianza ANOVA, uno para probar si hay preferencias por innovar en determinadas unidades dentro de una organización, y otro para probar si hay diferencias en la importancia entre los factores de innovación. El análisis ANOVA se basa en la descomposición de la variación total de los datos con respecto a la media global (SCT); a partir de la variación dentro de las muestras (SCD) o intra-grupos, cuantifica la dispersión de los valores de cada muestra con respecto a sus correspondientes medias. Finalmente, se efectúa un análisis de regresión lineal múltiple para verificar si algunos factores de innovación pueden ser explicados por otros factores que favorecen la innovación. A modo de resumen, la Tabla 4 presenta la ficha técnica del estudio.

\section{Resultados}

\section{Características de las empresas participantes}

En general, el 66.6\% empresas evaluadas superan los 15 años operando en el sector, luego, sus percepciones tienen un peso relevante para el presente estudio. En cuanto al nivel de ventas, cada una de ellas factura un promedio de US\$ 2.4 millones anuales, con un promedio de 170
Tabla 4: Ficha técnica del estudio

\begin{tabular}{|l|l|}
\hline Criterio & \multicolumn{1}{|c|}{ Acción } \\
\hline Población & $\begin{array}{l}\text { Empresas del sector construcción de } \\
\text { la región de Atacama, Chile }\end{array}$ \\
\hline Variables de medición & Relacionadas con la innovación \\
\hline Selección de la muestra & $\begin{array}{l}\text { Por conveniencia, socios de la } \\
\text { Cámara Chilena de la Construcción }\end{array}$ \\
\hline Tamaño de muestra & $\begin{array}{l}\text { 12 expertos, representantes de } \\
\text { empresas }\end{array}$ \\
\hline Composición de la muestra & $\begin{array}{l}\text { Directivos expertos de empresas } \\
\text { PYME del sector construcción }\end{array}$ \\
\hline Nivel de confianza y error & Se usó muestreo por conveniencia \\
\hline $\begin{array}{l}\text { Técnica recopilación de } \\
\text { datos }\end{array}$ & $\begin{array}{l}\text { Entrevistas y cuestionarios, escala } \\
\text { de Likert }\end{array}$ \\
\hline Periodo de registro de datos & Diciembre 2019 - Enero 2020 \\
\hline $\begin{array}{l}\text { Herramienta de registro y } \\
\text { análisis }\end{array}$ & SPSS Statistics V25 y MS-Excel \\
\hline Tipo de análisis estadístico & $\begin{array}{l}\text { Descriptivo, correlacional, análisis } \\
\text { de varianza ANOVA, y regresión } \\
\text { lineal múltiple }\end{array}$ \\
\hline
\end{tabular}

trabajadores. Desde el punto de vista organizacional, los expertos tienen una profesión técnica o universitaria, de los cuales el $33.3 \%$ posee cursos de postgrado. Asimismo, se midió la relación entre número de trabajadores y ventas promedio anuales, el coeficiente de correlación de Pearson entregó una alta correlación positiva $(\mathrm{R}=0.85)$, lo cual parece natural porque a mayor cantidad de trabajadores, mayores deben ser las ventas de las empresas.

\section{Relevancia de la innovación}

Usando una escala de Likert, se evaluó el grado de acuerdo sobre la afirmación "la innovación es una herramienta estratégica muy relevante para mejorar la competitividad de las empresas constructoras". Los resultados indicaron que el $83.3 \%$ de los expertos respondieron la alternativa MA y el $16.7 \%$ coincidió con la alternativa A. Para los grados MB, B, M las preferencias fueron nulas. Estos resultados confirman la relevancia que puede tener para la empresa constructora la innovación como una herramienta estratégica.

\section{Análisis de áreas pertinentes para innovar Calificación de áreas de innovación}

Tal como lo indica la Tabla 2 se establecieron siete unidades factibles para innovar dentro de una organización del sector de la construcción. Luego, con el fin de conocer 
cuáles son las unidades más pertinentes para la instalación de la innovación en empresas del sector construcción, se solicitó a los expertos que calificaran o valoraran estas siete unidades. Posteriormente, utilizando una escala de Likert donde se asigna 5 puntos a un área de Muy Alta pertinencia para innovar y, 1 punto para una de Muy Baja pertinencia. Los resultados obtenidos se presentan en Figura 1.

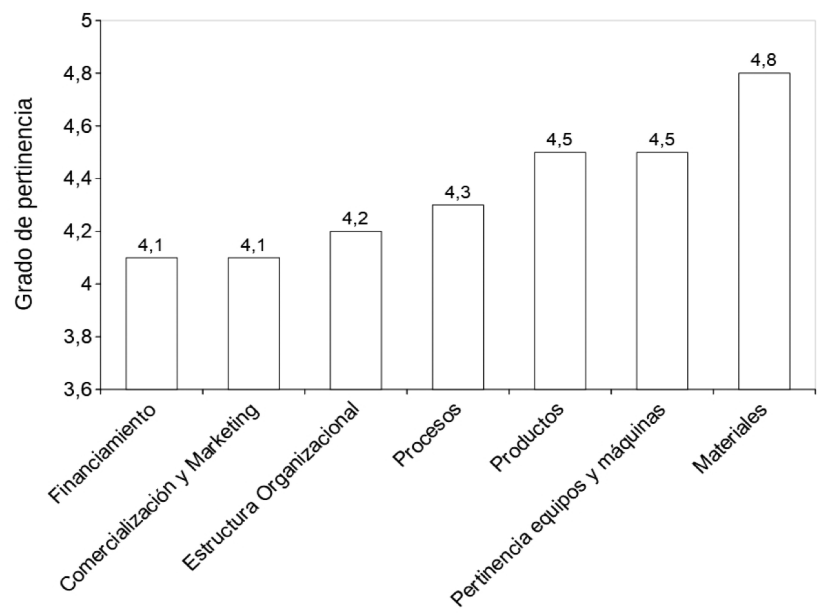

Figura 1: Resultados del grado de pertinencia para innovar

En la Figura 1, se puede observar que la valoración promedio más alta la obtuvo la unidad de materiales con 4.8, así como también equipos y máquinas y en productos, ambas con 4.5. Se calificó a las áreas financiamiento, comercialización y marketing (4.1) y a estructura organizacional (4.2), levemente por debajo al área procesos (4.3).

\section{Análisis correlacional de las unidades para innovar}

Con el propósito de medir el grado de relación entre las siete unidades factibles de innovar mostradas en el apartado anterior y percibida por los expertos, se efectuó un análisis de correlación. Los resultados obtenidos se presentan en la Tabla 5 y se muestra que la correlación positiva más alta se presentó entre las variables procesos y estructura organizacional con $r=0.84$. La evidencia empírica indicó que estas dos unidades están fuertemente relacionadas. Por otra parte, existe una relación negativa entre materiales y procesos, con $r=-0.33$; es decir, los expertos que privilegian innovar en materiales no piensan lo mismo respecto de innovar en procesos.

\section{Análisis de ANOVA de unidades de innovación}

También se efectuó un análisis de varianza ANOVA de
Tabla 5: Matriz de correlación entre las unidades de innovación

\begin{tabular}{|c|c|c|c|c|c|c|c|c|}
\hline \multicolumn{9}{|c|}{ Unidades de innovación } \\
\hline \multirow{8}{*}{ 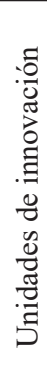 } & & 1 & 2 & 3 & 4 & 5 & 6 & 7 \\
\hline & 1 & 1.00 & & & & & & \\
\hline & 2 & -0.12 & 1.00 & & & & & \\
\hline & 3 & -0.33 & 0.58 & 1.00 & & & & \\
\hline & 4 & 0.84 & 0.00 & -0.11 & 1.00 & & & \\
\hline & 5 & 0.12 & 0.00 & 0.19 & 0.00 & 1.00 & & \\
\hline & 6 & 0.37 & 0.10 & 0.06 & 0.52 & -0.29 & 1.00 & \\
\hline & 7 & 0.27 & 0.11 & 0.57 & 0.47 & 0.11 & 0.63 & 1.00 \\
\hline
\end{tabular}

1: procesos, 2: productos, 3: materiales, 4: estructura organizacional, 5: máquinas y equipos, 6 : financiamiento, 7 : comercialización y marketing

un factor para ver si existía una diferencia significativa en las valoraciones de las siete unidades factibles de innovar. Se empleó un nivel de confianza de 95\%, y $\alpha=0.05$. La prueba de hipótesis es:

Hipótesis nula: las valoraciones de las unidades para innovar son similares.

Hipótesis alternativa: las valoraciones de las unidades para innovar no son similares.

Los resultados del análisis estadístico ANOVA entregó un $\mathrm{F}=1.49$ con probabilidad 0.19 , y un valor crítico para $\mathrm{F}$ de 2.21. Luego, se puede observar que $\mathrm{F}$ empírico $=1.49$ es menor al valor crítico $=2.21$. Luego, como $F$ se encuentra en zona de no rechazo, se cumple la hipótesis nula, es decir, todas las unidades son similares para innovar. No hay evidencia para comprobar que existen diferencias significativas en las valoraciones de las unidades factibles para innovar.

\section{Análisis de factores que favorecen la innovación} Calificación de factores favorables

Tal como lo indica la Tabla 2, se establecieron once factores que podrían favorecer o facilitar la instalación de la innovación al interior de una empresa del sector construcción. Posteriormente, se solicitó a los expertos que valoraran los once factores. Usando una escala de Likert donde se asignó 5.0 puntos a un factor que favorece Muy Alto la innovación y 1.0 punto para uno que favorece Muy Bajo, los resultados obtenidos se presentan en Figura 2.

Considerando los resultados mejor valorados en la Figura 2, se observa que el factor más valorado para la 


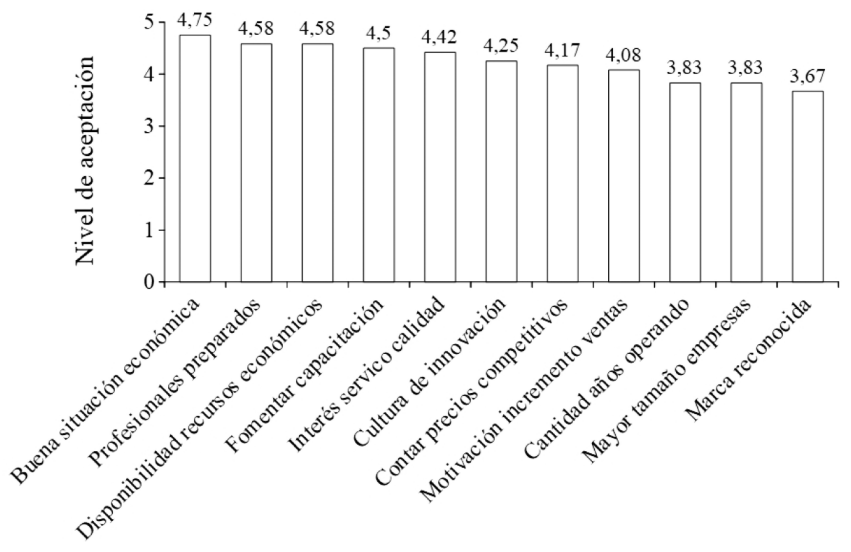

Figura 2: Resultados de factores que favorecen la innovación

instalación de la innovación en las empresas del sector construcción es la Buena situación económica, con un promedio de 4.75, es decir, en la medida que el país esté bien económicamente, por ejemplo, con crecimiento económico (PIB), una inflación controlada y una baja tasa de desempleo, entonces las personas tienden a comprar más y las empresas a innovar en la búsqueda de nuevos clientes. También se destacan como importantes los factores: Profesionales preparados (4.58) y Disponibilidad de recursos (4.58). Además, se puede apreciar que el cuarto factor en importancia apunta justamente a Fomentar la capacitación (4.50) de los trabajadores de las empresas del sector construcción.

\section{Análisis de ANOVA con factores favorables a la innovación}

Al igual que con las áreas factibles de innovar, se efectuó un análisis de varianza ANOVA para ver si existía una diferencia significativa en las valoraciones de los once factores favorables a la innovación. Se empleó un nivel de confianza de $95 \%$, y $\alpha=0.05$. La prueba de hipótesis es:

Hipótesis nula: las valoraciones de todos los factores son similares

Hipótesis alternativa: las valoraciones de todos los factores no son similares

El informe de resultados que entregó el software estadístico señaló $\mathrm{F}=2.78$ con probabilidad 0.0038 , y un valor crítico para F de 1.90 .

De los resultados se puede observar que, $\mathrm{F}$ empírico $=$ 2.78 es mayor al valor crítico $=1.90$; luego, como $\mathrm{F}$ se encuentra en zona de rechazo, se rechaza la hipótesis nula.
Por lo tanto, hay evidencia para comprobar que existen diferencias significativas en los factores favorables para innovar. Del análisis de calificaciones se observa que el factor Buena situación económica es el factor más favorable para la innovación.

\section{Análisis de regresión lineal múltiple con factores favorables a la innovación \\ Con los factores favorables a la innovación se buscó un modelo significativo del tipo mostrado en la fórmula (1).}

$\mathrm{Y}=\alpha+\beta 1 \mathrm{X} 1+\beta 2 \mathrm{X} 2+\beta 3 \mathrm{X} 3+\ldots+\beta \mathrm{Xi}$

Buscando potenciales modelos y coeficientes significativos con un nivel de significancia de 5\%, se sometió a prueba el modelo de la fórmula (2).

Marca reconocida $=\alpha+\beta 1$ (Tamaño empresa) $+\beta 2$ (Motivación por incrementar ventas)

La hipótesis es que el modelo es no lineal y los coeficientes son iguales a $0(\beta i=0)$. En la Tabla 6 se puede observar que la probabilidad asociada al estadístico $\mathrm{F}$ es 0.000049 y es mucho menor al nivel de significancia de 0.05 , luego, la hipótesis se rechaza, y el modelo es lineal.

Tabla 6: Resultados de análisis de ANOVA del modelo

\begin{tabular}{|l|c|c|c|c|c|}
\hline $\begin{array}{l}\text { Origen } \\
\text { de las } \\
\text { variaciones }\end{array}$ & $\begin{array}{c}\text { Suma } \\
\text { de los } \\
\text { cuadrados }\end{array}$ & $\begin{array}{c}\text { Grados } \\
\text { de } \\
\text { libertad }\end{array}$ & $\begin{array}{c}\text { Promedio } \\
\text { de los } \\
\text { cuadrados }\end{array}$ & F & $\begin{array}{c}\text { Valor } \\
\text { crítico } \\
\text { para F }\end{array}$ \\
\hline Regresión & 13.047 & 2 & 6.523 & 36.25 & 0.000049 \\
\hline Residuos & 1.6195 & 9 & 0.180 & - & - \\
\hline Total & 14.667 & 11 & - & - & - \\
\hline
\end{tabular}

De la Tabla 7 se puede observar los coeficientes del modelo y sus probabilidades asociadas, todas ellas son menores a 0.05 , luego, son significativas. Además, como el indicador Durbin-Watson fue de 1.86 entonces los residuos son independientes, y si se asume normalidad y homocedasticidad entonces el modelo es lineal y queda representado por la fórmula (3).

Marca reconocida $=-2.1576+0.5815($ Tamaño empresa $)+$ 0.8804 (Motivación por incrementar ventas) 
Tabla 7: Resultados de análisis de coeficientes del modelo

\begin{tabular}{|l|c|c|c|c|}
\hline & Coeficientes & $\begin{array}{c}\text { Error } \\
\text { típico }\end{array}$ & Estadístico t & Probabilidad \\
\hline Intercepción & -2.1576 & 0.7945 & -2.7155 & 0.0238 \\
\hline Variable X1 & 0.5815 & 0.1698 & 3.4236 & 0.0076 \\
\hline Variable X2 & 0.8804 & 0.2616 & 3.3649 & 0.0083 \\
\hline
\end{tabular}

Se concluye que tener una marca reconocida depende en forma lineal y positiva del tamaño de la empresa y de la motivación que tenga esa misma organización por incrementar las ventas.

\section{Análisis de factores que desfavorecen la innovación}

Finalmente, se solicitó a los expertos que identificaran los tres factores más desfavorables para la instalación de la innovación en empresas del sector construcción. Los factores que desfavorecen la innovación en las empresas consultadas son mostrados en la Tabla 8 .

Tabla 8: Factores más desfavorables para la instalación de la innovación en empresas del sector construcción (en \%)

\begin{tabular}{|l|c|c|c|}
\hline $\begin{array}{l}\text { Falta de cultura de } \\
\text { innovación }\end{array}$ & 77.8 & $\begin{array}{c}\text { Poca motivación de } \\
\text { directivos }\end{array}$ & 22.2 \\
\hline $\begin{array}{l}\text { Estructura organizacional } \\
\text { poco flexible }\end{array}$ & 66.7 & $\begin{array}{c}\text { Falta de confianza de } \\
\text { directivos }\end{array}$ & 11.1 \\
\hline Escasez de recursos & 55.6 & $\begin{array}{c}\text { Falta de } \\
\text { financiamiento } \\
\text { externo }\end{array}$ & 11.1 \\
\hline Baja capacidad gerencial & 55.6 & \multicolumn{2}{|l}{} \\
\cline { 1 - 4 }
\end{tabular}

A partir de los datos de la Tabla 8, se puede destacar que el factor más mencionado que desfavorece la instalación de la innovación es Falta de cultura de innovación, identificado por el $77.8 \%$ de los expertos. Estos últimos piensan que la existencia de una cultura de innovación organizacional es fundamental para incorporar la innovación en las distintas áreas de una empresa, y por cierto, una falta de cultura desfavorece la innovación. El segundo factor más desfavorable es Estructura organizacional poco flexible. Esto puede significar que para facilitar la incorporación de la innovación, las organizaciones deben ser flexibles y adecuarse con rapidez a los cambios, ya sean internos o externos. Por ejemplo, en la conformación de equipos de trabajo multidisciplinarios para enfrentar desafíos imprevistos del entorno, o en el uso de un nuevo material en la construcción que ha incorporado recientemente la competencia. En tercer lugar figura la Escasez de recursos y la Baja capacidad gerencial.

\section{Análisis y discusión}

Como se ha visto, el $83.3 \%$ de los expertos encuestados está "muy de acuerdo" en que "la innovación es una herramienta muy relevante para mejorar la competitividad de las empresas constructoras", y el 16.7\% está "de acuerdo" con la misma proposición. Luego, se comprueba la relevancia de la innovación como herramienta estratégica. Esto último, es coincidente con un estudio realizado a Mipymes de la construcción en Nigeria concluyendo que la innovación tiene una influencia positiva en el desarrollo de la empresa constructora (Adamu et al., 2020). En las unidades factibles de innovar, la valoración promedio más alta la obtuvo la unidad de Materiales con 4.75. Esto significa que los expertos estiman que en ella se podría innovar con mayor facilidad, probablemente incorporando nuevos materiales y de mayor calidad. También se podría innovar en Productos (4.5) y en Equipos y máquinas (4.5). Del análisis de correlación entre las unidades factibles de innovar, la correlación más fuerte positiva, con $r=$ 0.84, se presenta entre las unidades Procesos y Estructura organizacional, lo cual tiene similitud con un estudio realizado en pequeñas empresas constructoras de la ciudad de Puebla en México (Martínez et al., 2014), concluyendo que los administradores consideran la unidad de procesos como la más importante para la innovación. Por otra parte, existe una relación negativa entre Materiales y Procesos, con $r=-0.33$; es decir, los expertos que privilegian innovar en materiales no piensan lo mismo respecto de innovar en procesos. Respecto del análisis ANOVA entre las unidades, con un nivel de significancia de $95 \%$ y $\alpha=0.05$, no hay evidencia para comprobar que existen diferencias significativas en las calificaciones entre las unidades factibles de innovar.

Con respecto a los factores que favorecen la instalación de la innovación, el factor mejor calificado es la "Buena situación económica", con un promedio de 4.75. También se destacan como importantes los factores: Disponibilidad de recursos (4.58) y Profesionales preparados (4.58). Del análisis ANOVA, se puede observar que hay evidencia para comprobar que existen diferencias significativas en los factores favorables para innovar. Asimismo, otro 
estudio para el sector europeo de la construcción (Barata y Fontainha, 2017), determinó que el sector sí innova, y los factores que más contribuyen a esta innovación son los proveedores y el crecimiento de la empresa. El mismo estudio, concluyó que el tamaño de la empresa es más relevante para la innovación de procesos que para la innovación del producto, y las empresas que participan en los mercados internacionales innovan más que las focalizadas en los mercados locales y regionales.

En relación a los factores que desfavorecen la instalación de la innovación, el factor más mencionado es la Falta de cultura de innovación, identificado por el $77.8 \%$ de los expertos. Esto significa que ellos piensan que la existencia de una cultura de innovación organizacional es fundamental para incorporar la innovación en las distintas áreas de una empresa. El segundo factor más desfavorable es Estructura organizacional poco flexible, con el $66.7 \%$ de las menciones. Esto puede significar que para facilitar la incorporación de la innovación las organizaciones deben ser flexibles y adecuarse con rapidez a los cambios, ya sean internos o externos. Por ejemplo, en la conformación de equipos de trabajo multidisciplinarios para enfrentar desafíos imprevistos del entorno, o en el uso de un nuevo material en la construcción que ha incorporado recientemente la competencia. En tercer lugar figura la Escasez de recursos y la Baja capacidad gerencial, ambas con el $55.6 \%$ de las menciones. A nivel internacional, se ha visto que cada empresa constructora vive una realidad diferente, por ejemplo, un estudio realizado a empresas constructoras nigerianas (Abdu y Jibir, 2018), el principal factor que desfavorece la instalación de la innovación es la edad de sus empleados y el nivel de educación. Por otra parte, un estudio realizado a Pymes de la construcción en Kuwait (Alzougool, 2019), concluyó que la innovación se plantea en 5 dimensiones: organización, proceso, producto, cultura y recursos para ser exitosos. Estas dimensiones, son coincidente con la realidad de las empresas constructoras de la región de Atacama, que han demandado mayor intervención en estas áreas puntuando en rango de entre 4.25 y 4.75 considerando un máximo de 5.0 de la escala de Likert, lo que orienta el camino para desarrollar estrategias futuras para la instalación de la innovación en la organización.

\section{Conclusiones}

En esta investigación se realizó una encuesta a empresarios y directivos de 12 empresas constructoras de la región de Atacama con el propósito de valorar los factores que pueden favorecer o desfavorecer la instalación de la innovación al interior de las empresas constructoras. Los resultados indicaron claramente que la totalidad de los expertos encuestados opina que la innovación es una herramienta estratégica muy relevante para mejorar la competitividad de las empresas constructoras de la región de Atacama.

Además, se determinó que las unidades más factibles para innovar dentro de una empresa del sector de la construcción son: materiales, productos, y equipos y máquinas. Por otra parte, los expertos privilegian fuertemente las unidades de procesos y estructura organizacional como base para la instalación de la innovación en el sector construcción. Por el contrario, los factores que no favorecen la instalación de la innovación, son en primer lugar, la falta de cultura de innovación, en segundo lugar la estructura organizacional poco flexible, y compartiendo el tercer lugar, la escasez de recursos y la baja capacidad gerencial.

\section{Agradecimientos}

Los autores agradecen a la gerencia y a los asociados de la Cámara Chilena de la Construcción de Atacama, por su valiosa colaboración en el desarrollo de este estudio.

\section{Referencias}

Abdel-Razek, R.H. (1998). Factors affecting construction quality in Egypt: identification and relative importance. Engineering, Construction and Architectural Management 5(3), 220-227

Abdu, M. and Jibir, A. (2018). Determinants of firms innovation in Nigeria. Kasetsart Journal of Social Sciences 39(3), 448-456

Adamu, U.G., Hussin, S.R. and Ismail, N.A. (2020). Effect of marketing innovation on performance of small and medium enterprises in Nigeria. International Journal of Innovation, Creativity and Change 11(12), 353-370

Alzougool, B. (2019). The innovativeness of small and medium enterprises in Kuwait. Journal of Advanced Management Science 7(4), 142-147

Aouad, G.F., Ozorhon, B. and Abbott, C. (2010). Facilitating innovation in construction: directions and implications for research and policy. Construction Innovation 10(4), 374-394 
Arévalo, D.X. and Padilla, C. (2016). Medición de la confiabilidad del aprendizaje del programa RStudio mediante Alfa de Cronbach. Revista Politécnica 37(1), 68-75

Barata, J.M. and Fontainha, E. (2017). Determinants of innovation in European construction firms. Technological and Economic Development of Economy 23(6), 915-936

BCN (2013). https://www.bcn.cl/leyfacil/recurso/como-crearuna-pyme $(22 / 05 / 2021)$

Blayse, A.M. and Manley, K. (2004). Key influences on construction innovation. Construction Innovation 4(3), 143-154

Bygballe, L.E. and Ingemansson, M. (2014). The logic of innovation in construction. Industrial Marketing Management 43(3), 512-524

CDT (2010). Construyendo innovación. Corporación de Desarrollo Tecnológico, Cámara Chilena de la Construcción, Santiago de Chile, http://www.otcchile.cl/uploads/library/2010 gestion_innovacion_20190320194152_64449.pdf(16/05/2020)

CDT (2012). Gestión de la innovación en la construcción. Guía metodológica. Corporación de Desarrollo Tecnológico, Cámara Chilena de la Construcción. Santiago de Chile, https://www.cdt. cl/category/publicaciones-cdt/ (16/05/2020)

Cheung, S.O., Suen, H.C. and Cheung, K.K. (2004). PPMS: a web-based construction project performance monitoring system. Automation in Construction 13(3), 361-376

Cleasby, B. (2004). Consolidation events: learning and capability building in project-based firms. University of Sussex, Brighton, UK

CORFO (2017). Glosario de conceptos corporativos. Corporación de Fomento de la Producción de Chile. Santiago de Chile

Dikmen, I., Birgonul, M.T. and Artuk, S.U. (2005). Integrated framework to investigate value innovations. Journal of Management in Engineering 21(2), 81-90

DTI (2007). Innovation in services. Occasional paper No. 9. Department of Trade and Industry, London, UK

Dubois, A. and Gadde, L.E. (2002). The construction industry as a loosely coupled system: implications for productivity and innovation. Construction Management and Economics 20(7), $621-31$

EC (2010). Lisbon strategy evaluation document. Commission staff working document, Brussels, Belgium
Gambatese, J.A. and Hallowell, M. (2011). Factors that influence the development and diffusion of technical innovations in the construction industry. Construction Management and Economics 29(5), 507-517

Gledson, B.J. and Phoenix, C. (2017). Exploring organisational attributes affecting the innovativeness of UK SMEs. Construction Innovation 17(2), 224-243

GII (2019). Indicator rankings \& analysis. Global innovation index https://www.globalinnovationindex.org/analysis-indicator $(16 / 05 / 2020)$

Goodrum, P.M. and Haas, C.T. (2000). Variables affecting innovations in the US construction industry. Construction Congress VI: Building Together for a Better Tomorrow in an Increasingly Complex World, ASCE, K.D. Walsh (ed.), Florida, USA, 525-533

Hardie, M. and Newell, G. (2011). Factors influencing technical innovation in construction SMEs: an Australian perspective. Engineering, Construction and Architectural Management 18(6), 618-636

Heimonen, T. (2012). What are the factors that affect innovation in growing SMEs?. European Journal of Innovation Management 15(1), 122-144

Heitor, M., Horta, H., Castañón, R., Sbragia, R. and Jiménez, A. (2014). Can Latin America move forward after a lost decade in technical change?: Looking at opportunities for knowledgebased change in times of increasing uncertainty. Journal of Technology Management \& Innovation 9(4), 1-19

Hölzl, W. (2009). Is the R\&D behaviour of fast-growing SMEs different? Evidence from CIS data for 16 countries. Small Business Economics 33(1), 59-75

Hoogstra, G.J. and van Dijk, J. (2004). Explaining firm employment growth: does location matter?. Small Business Economics 22(3), 179-192

Koskela, L. and Vrijhoef, R. (2001). Is the current theory of construction a hindrance to innovation?. Building Research \& Information 29(3), 197-207

Littunen, H. and Tohmo, T. (2003). The high growth in new metal-based manufacturing and business service firms in Finland. Small Business Economics 21(2), 187-200 
Luu, T.V., Kim S.Y., Cao, H.L. and Park, Y.M. (2008). Performance measurement of construction firms in developing countries. Construction Management and Economics 26(4), 373-386

Manley, K. and Mcfallan, S. (2006). Exploring the drivers of firm-level innovation in the construction industry. Construction Management and Economics 24(9), 911-920

Martínez, R.M., Vera, M.A. and Vera, J.G.S. (2014). Cultura de la innovación en las pequeñas empresas constructoras de Puebla, México. Revista Global de Negocios 2(3), 91-100

Mendonça, S., Pereira, A.S. and Godinho, C.M. (2004). Trademarks as an indicator of innovation and industrial change. Research Policy 33(9), 1385-1404

North, D. and Smallbone, D. (2000). The innovativeness and growth of rural SMEs during 1990s. Regional Studies 34(2), $145-157$

OECD (2005). Oslo manual. The measurement of scientific and technological activities. Guidelines for collecting and interpreting innovation data. $3^{\text {rd }}$ ed., Organisation for Economic Co-operation and Development, Paris, France

Seaden, G. and Manseau, A. (2001). Public policy and construction innovation. Building Research \& Information 29(3), 182-196

Sexton, M.G. and Barrett, P.S. (2003). A literature synthesis of innovation in small construction firms: insights, ambiguities and questions. Construction Management and Economics 21(6), 613-622

Slaughter, E.S. (2000). Implementation of construction innovations. Building Research \& Information 28(1), 2-17

Talebi, K., Ghavamipour, M. and Irandust, A. (2012). Innovation in Irans small and medium size enterprises (SMEs): Prioritize influence factors affecting innovation of SMEs, using analytic network process (ANP) method. African Journal of Business Management 6(43), 10775-10785

Talukder, M. (2012). Factors affecting the adoption of technological innovation by individual employees: an Australian study. Procedia-Social and Behavioral Sciences 40, 52-57

Winch, G.M. (2003). How innovative is construction? Comparing aggregated data on construction innovation and other sectors - a case of apples and pears. Construction Management and Economics 21(6), 651-654

\section{Anexo}

Cuestionario

Proyecto de Investigacion

Medición de Innovación en Empresas del Sector Construcción

En la Región de Atacama

PARTE A: Datos de la empresa y equipo directivo

Maque con una X la alternativa de su preferencia.

1.- Dentro del sector de la construcción, ¿cuál es el principal campo de especialización de la empresa?

Edificación y vivienda

Infraestructura y obras civiles

Urbanización

Obras menores de la construcción

Otro:

2.- ¿Cuántos años de operación en el sector construcción tiene la empresa?

Menos de 3 años

Entre 4 y 6 años

Entre 7 y 10 años

Entre 11 y 15 años

Más de 15 años

3.- ¿En qué rango de ventas anuales se encuentra la empresa constructora?

Menos de UF 2.400 anuales

Entre UF 2.401 a UF 25.000 anuales

Entre UF 25.001 a UF 100.000 anuales

Más de UF 100.000 anuales

4.- ¿Con cuántos trabajadores de planta $\mathrm{y}$ en forma temporal, en promedio, opera la empresa constructora?

Entre 1 y 5 trabajadores

Entre 6 y 50 trabajadores

Entre 51 y 400 trabajadores

Más de 400 trabajadores

5.- ¿Qué tipo de formación profesional tiene el Socio Principal o Gerente General de la empresa?

Técnica ( $2-3$ años)

Universitaria (4 - 5 años)

Postgrado (6 o más)

Otro:

6.- ¿Qué profesión posee el Socio Principal o Gerente General de la empresa?

Técnico en Construcción

Constructor Civil

Ingeniero en Construcción

Arquitecto

Ingeniero Comercial

Ingeniero Civil

Otro: 
PARTE B: Medición de variables de innovación:

Respecto de las afirmaciones que se plantean en las siguientes tablas, indique su grado de acuerdo con ellas marcando una $\mathrm{X}$ en la columna de su preferencia, según la siguiente escala:

MB: $\quad$ Muy bajo acuerdo

A: $\quad$ Alto acuerdo

B: $\quad$ Bajo acuerdo

MA: Muy alto acuerdo

M: $\quad$ Mediano acuerdo

\section{UNIDADES PERTINENTES PARA INNOVAR}

\begin{tabular}{|c|l|l|l|l|l|l|}
\hline$N^{\circ}$ & \multicolumn{1}{|c|}{ Unidades } & MB & B & M & A & MA \\
\hline 1 & $\begin{array}{l}\text { La innovación es una herramienta } \\
\text { estratégica muy relevante para } \\
\text { mejorar la competitividad de las } \\
\text { empresas constructoras. }\end{array}$ & & & & \\
\hline 2 & $\begin{array}{l}\text { El área de procesos en la construcción } \\
\text { es muy pertinente para innovar. }\end{array}$ & & & & \\
\hline 3 & $\begin{array}{l}\text { El área de productos en la } \\
\text { construcción es muy pertinente para } \\
\text { innovar }\end{array}$ & & & & & \\
\hline 4 & $\begin{array}{l}\text { El área de materiales en la } \\
\text { construcción es muy pertinente para } \\
\text { innovar. }\end{array}$ & & & & & \\
\hline 5 & $\begin{array}{l}\text { El área de estructura organizacional } \\
\text { y personal es muy pertinente para } \\
\text { innovar. }\end{array}$ & & & & & \\
\hline 6 & $\begin{array}{l}\text { El área de equipamiento maquinaria } \\
\text { es muy pertinente para innovar. }\end{array}$ & & & & \\
\hline 7 & $\begin{array}{l}\text { El área de financiamiento para la } \\
\text { construcción es muy pertinente para } \\
\text { innovar. }\end{array}$ & & & & & \\
\hline 8 & $\begin{array}{l}\text { El área de comercialización y } \\
\text { marketing es muy pertinente para } \\
\text { innovar. }\end{array}$ & & & & \\
\hline
\end{tabular}

\section{FACTORES QUE FAVORECEN LA INNOVACIÓN}

\begin{tabular}{|c|c|c|c|c|c|c|}
\hline $\mathrm{N}^{\circ}$ & Factores & MB & B & M & A & MA \\
\hline 1 & $\begin{array}{l}\text { Una buena situación económica } \\
\text { en el país, con crecimiento y bajo } \\
\text { desempleo favorece la innovación. }\end{array}$ & & & & & \\
\hline 2 & $\begin{array}{l}\text { El interés de la empresa constructora } \\
\text { por entregar un servicio o producto } \\
\text { de calidad favorece la innovación. }\end{array}$ & & & & & \\
\hline 3 & $\begin{array}{l}\text { Contar con precios competitivos y } \\
\text { atractivos para los clientes favorece } \\
\text { la innovación. }\end{array}$ & & & & & \\
\hline 4 & $\begin{array}{l}\text { Una política o cultura interna de } \\
\text { innovación claramente establecida y } \\
\text { difundida por la empresa constructora } \\
\text { favorece la innovación. }\end{array}$ & & & & & \\
\hline 5 & $\begin{array}{l}\text { El contar con gerentes, profesionales } \\
\text { y especialistas preparados para la } \\
\text { innovación favorece la innovación. }\end{array}$ & & & & & \\
\hline 6 & $\begin{array}{l}\text { La disponibilidad de } \\
\text { económicos recursos } \\
\text { investigación y desarrollo favorece } \\
\text { la innovación. }\end{array}$ & & & & & \\
\hline 7 & $\begin{array}{l}\text { Fomentar un plan de capacitación y } \\
\text { desarrollo del personal en materias } \\
\text { orientadas a la innovación favorece } \\
\text { la innovación. }\end{array}$ & & & & & \\
\hline 8 & $\begin{array}{l}\text { La motivación por incrementar las } \\
\text { ventas y las ganancias de la empresa } \\
\text { constructora favorece la innovación. }\end{array}$ & & & & & \\
\hline
\end{tabular}

\begin{tabular}{|l|l|l|l|l|l|l|}
\hline 9 & $\begin{array}{l}\text { Una importante cantidad de años } \\
\text { operando en el sector construcción } \\
\text { favorece la innovación. }\end{array}$ & & & & & \\
\hline 10 & $\begin{array}{l}\text { Contar con una marca empresarial } \\
\text { reconocida y posicionada en el } \\
\text { mercado favorece la innovación. }\end{array}$ & & & & & \\
\hline 11 & $\begin{array}{l}\text { El mayor tamaño de las empresas } \\
\text { constructoras, medido en volúmenes } \\
\text { de ventas, favorece la innovación. }\end{array}$ & & & & & \\
\hline
\end{tabular}

A continuación, de la lista siguiente de $\mathbf{7}$ factores seleccione $\mathbf{3}$ factores que NO FAVORECEN LA INNOVACIÓN y efectúe un ranking con esos 3 factores escogidos. En la columna RANKING coloque un 1 al factor que menos favorece la innovación, luego, un 2 al segundo factor que menos favorece y, finalmente, un 3 al tercer factor que menos favorece la innovación.

\begin{tabular}{|c|l|c|}
\hline $\mathrm{N}^{\circ}$ & Factor & RANKING \\
\hline 1 & $\begin{array}{l}\text { Falta de capacidad gerencial y profesional en la } \\
\text { constructora para innovar. }\end{array}$ & \\
\hline 2 & Escasez de recursos económicos para innovar. & \\
\hline 3 & Falta de confianza en el personal de la constructora. & \\
\hline 4 & $\begin{array}{l}\text { Tener una estructura organizacional poco flexible } \\
\text { a los cambios. }\end{array}$ & \\
\hline 5 & $\begin{array}{l}\text { Poca motivación de equipo directivo por mejorar } \\
\text { los procesos, productos y servicios entregados al } \\
\text { cliente. }\end{array}$ & \\
\hline 6 & $\begin{array}{l}\text { Falta de una política y cultura de innovación } \\
\text { interna en la empresa constructora. }\end{array}$ & \\
\hline 7 & $\begin{array}{l}\text { Dificultad para lograr financiamiento externo de } \\
\text { largo plazo para innovar. }\end{array}$ & \\
\hline
\end{tabular}

